\title{
Default cascades: When does risk diversification increase stability?
}

\author{
Stefano Battiston ${ }^{\mathrm{a}, *}$, Domenico Delli Gatti ${ }^{\mathrm{b}}$, Mauro Gallegati ${ }^{\mathrm{c}}$, Bruce Greenwald ${ }^{\mathrm{d}}$, Joseph E. Stiglitz ${ }^{\mathrm{d}}$ \\ a ETH Zurich, Switzerland \\ ${ }^{\mathrm{b}}$ Catholic Univ. of Milan, Italy \\ c Polit. Univ. of Marche, Ancona, Italy \\ d Columbia University, New York, USA
}

\section{A R T I C L E I N F O}

\section{Article history:}

Received 18 November 2010

Received in revised form 18 January 2012

Accepted 27 January 2012

Available online 8 March 2012

\section{JEL classification:}

D85

G01

G21

Keywords:

Systemic risk

Network models

Contagion

Financial crisis

\begin{abstract}
A B S T R A C T
We explore the dynamics of default cascades in a network of credit interlink-ages in which each agent is at the same time a borrower and a lender. When some counterparties of an agent default, the loss she experiences amounts to her total exposure to those counterparties. A possible conjecture in this context is that individual risk diversification across more numerous counterparties should make also systemic defaults less likely. We show that this view is not always true. In particular, the diversification of credit risk across many borrowers has ambiguous effects on systemic risk in the presence of mechanisms of loss amplifications such as in the presence of potential runs among the short-term lenders of the agents in the network.
\end{abstract}

(C) 2012 Elsevier B.V. All rights reserved.

\section{Introduction}

One of the most important issues that the Global Financial Crisis (GFC) has brought to the fore concerns the effects on systemic risk of the increasing interdependence both among the main actors of financial markets and among financial markets across countries. In particular, the notion of too-big-too-fail becomes more subtle, while the regulatory mechanisms based only on a bank's own risk may fail to mitigate aggregate risk-shifting incentives, and can, in fact, accentuate systemic risk (Acharya, 2009).

Increasing interdependence of global financial markets - mainly achieved by means of liberalization of capital flows - may be supposed to lead to greater worldwide financial stability, as risks are spread around the world. Increasing interdependence of economic agents, on the other hand, allows for a better diversification of individual risk, as risks are spread around the set of connected partners: The larger the number of borrowers a lender is connected to in a network of borrowing/lending relationship, the smaller the fraction of an idiosyncratic shock (which leads to the default of a borrower) the lender has to bear. This, other things being equal and assuming

\footnotetext{
* Corresponding author.

E-mail address: sbattiston@ethz.ch (S. Battiston).
}

idiosyncratic shocks are not correlated, i.e. they are not springing from the same source. It is reasonable to conjecture, therefore, that individual risk diversification leads to a lower systemic risk. There is at least one good and obvious reason to think that this is indeed the case. Consider a network of borrowing/lending relationships. Suppose agent $i$ lends 1 unit to each node in a neighborhood consisting of $k$ borrowers. When a borrower defaults (hence the idiosyncratic shock to $i$ ), the loss the lender experiences (due to the non performing loan) amounts to her relative exposure to the borrower. The relative loss amounts to $1 / k$. By increasing the number of counterparties so that chik tends asymptotically to infinity, the impact of a negative shock (the relative exposure to each borrower) tends to zero. Since the lender hit by an idiosyncratic shock does not feel the pinch and does not react to it, there will not be further repercussions of the shock itself. In this case, we can rule out domino effects and default cascades. Hence enhanced risk diversification through increasing network density reduces systemic risk.

The GFC has cast doubt on these conclusions. The breakdown of a relatively small segment of the US financial system has not only spread to the other segments - an obvious consequence of interdependence - but has also pushed the system on the verge of a "financial meltdown" at the time of the Lehman Brothers bankruptcy. Moreover, this event has triggered a financial crisis worldwide due to capital market integration. One legitimate 
conjecture therefore, is that increasing interdependence of agents and integration of financial markets in principle may not reduce but increase the risk of a systemic collapse.

Empirical research aimed at estimating systemic risk before the GFC found very little evidence of global vulnerability (Bartram et al., 2007), confirming the view that risk diversification had been pushed so far as to reduce systemic risk to a negligible level. A remarkable body of empirical literature on stress-testing in financial systems also confirmed the view, stating that the default of an individual institution was typically not able to trigger a domino effect (see Elsinger et al., 2006; Boss et al., 2004; Furfine, 2003). The empirical evidence accumulated during the GFC, however, has raised legitimate doubts on the adequacy of the procedure adopted to carry out these stress-tests (Haldane, 2009; Amini et al., 2010). The unraveling of the GFC has overwhelmingly shown that systemic risk is not negligible and domino effects are likely despite the recent impressive increase of risk diversification (Brunnermeier, 2008).

According to the theoretical literature, Allen and Gale (2000) is the most important contribution to the analysis of "financial contagion" through credit interlinkages among banks. They show that, given full diversification of risk at the level of the individual bank, the spread of an unexpected liquidity shock and its systemic effects depend crucially on the pattern of interconnectedness among banks. When the network is complete - i.e. density is at its maximum - and the amount of interbank deposits held by each bank is evenly spread over all other banks, the impact of the shock is easily mitigated. When the network is connected but incomplete, with banks only having few counterparties, the system is more fragile. When the incomplete network assumes the typical structure of a "wheel" or a "cycle", the shock may lead to a systemic collapse. In this case, in fact, the shock is toppling one bank after the other along the network cycle. In the end, therefore, given full diversification of (individual) risk, a complete network is more resilient than an incomplete one.

A recent, post-crisis strand of literature has tried to identify the conditions upon which an increase of network density - i.e. a scenario in which the topology of the network tends toward completeness - is not beneficial, i.e. does not reduce systemic risk (see Battiston et al., 2009; Stiglitz, 2010; Castiglionesi and Navarro, 2010; Allen et al., 2010; Wagner, 2010). In the present paper we contribute to this new line of research by exploring the mechanisms that, following the default of an agent, may lead to an increase of systemic risk when connectivity increases. Our approach is related to the framework put forward by (Eisenberg and Noe, 2001) in order to analyze the effects of an agent's default on the cash flows of the counterparties. Such framework has been further studied also in Gai and Kapadia (2010a) and in Cont et al. (2010), where the default of a bank decreases the value of the assets of each counterparty in the interbank market. In this approach, the representation of the agents in the credit network is stylized and based on accounting identities. Behavioural assumptions are kept to a minimum: agents neither choose their capital structure (and thus their level of financial robustness), nor the partners to be connected to. Moreover, agents do not interact strategically. This static balance sheet approach - similar in spirit to the procedure adopted to carry out stress tests on banks - may look somehow mechanical (Christian and Upper, 2011) but allows to characterize analytically the emergence of systemic risk as function of essentially two determinants: i) the fraction of defaulting counterparties of each agent and ii) the initial financial robustness of each agent (Gai and Kapadia, 2010a).

In our paper, we model a network of borrowing/lending relationships among financial institutions ("banks"). These institutions are also active on "financial markets", i.e. they trade financial obligations with agents outside the network itself. For instance, they can collect deposits from households or get short-term loans from outside investors. Each agent is represented by a stylized balance sheet. Balance sheets are interrelated, as the asset of one agent (lending bank) is a liability for another agent (borrowing bank). The intertwined dynamics of the individual equity ratios are the driving force of the change in the credit network. In particular we will focus on changes produced by borrowers' defaults, which weaken the financial robustness of lenders and may therefore induce further defaults. In this context, therefore, from the initial default of one or few agents may endogenously follow the default of some other agents in a full-fledged default cascade.

In a nutshell, we carry out the following exercise. We assume an initial allocation of assets and liabilities across agents and an initial set of defaults. We then derive a law of motion for the financial robustness - as measured by the equity ratio - of the agents concerned by the default of one or more counterparties. Finally, we investigate how the size of the default cascade is affected by the initial distribution of robustness and by the level of risk diversification in the network.

The core feature of our model of the credit network is the fact that balance sheets are interrelated, and therefore the dynamics of the individual equity ratios are intertwined. This fact is the source of the externalities which play a crucial role in the model. We introduce a distinction between two types of externalities which correspond to different properties in relations to systemic risk. With the first type, the default of an agent (borrower) has an obvious and immediate effect on the financial robustness of its counterparties (lenders) in a credit network. The non-performing loan, in fact, translates into a reduction of the lender's equity. However, there are no further effects of the default on the counterparties. Whenever the market value of total assets in the counterparty's portfolio becomes smaller than that of liabilities, the counterparty in turn defaults. If some other counterparties in turn, default on their counterparties a cascade of defaults may ensue.

In this baseline scenario, which we label as external effect of the first type, we find different regimes, in which increasing connectivity may have a beneficial role or a detrimental one (or no role at all). When financial robustness is not very different across agents (the degree of heterogeneity and therefore the variance of equity ratios is relatively small), increasing connectivity makes the system more resilient to systemic defaults. More precisely, with increasing connectivity the system remains stable even at lower values of average robustness. On the other hand, increasing network density, may stimulate systemic defaults when: the initial robustness is heterogeneous across agents (high variance), but the average robustness is low and there is an initial large enough shock. The reason why, from a systemic point of view, in such a situation it is better to concentrate risk instead of diversifying it is that spreading the losses make more agents default (since are already fragile).

We also model an external effect of the second type which, in contrast to the first type, involves an amplification of losses along the chain of lending relations. The ambiguous role of diversification on systemic risk is in this case much more pronounced. We suspect this second mechanism to appear in several situations, but in this paper we focus on one specific case. Namely, we show how the mechanism arises if, in addition to the ingredients of the baseline model, we assume that agents borrow also short-term and are exposed to the potential run of the short-term lenders. When the agent is hit by the default of one or more of her counterparties (for brevity, the initial default), her short-term creditors cannot rule out that other counterparties may default, because they do not know with certainty the situation of the counterparties. This means that the chances that the agent defaults have increased, although technically she is still solvent. As a result, short-term creditors have to decide whether to roll-over debt to the agent or not, taking into 
account that the other creditors do the same reasoning. In other words, the second type of externalities may arise when considerations of illiquidity enter into the picture, in the presence of imperfect information.

For sake of simplicity, we do not model the coordination game of the short-term creditors and we follow instead a reduced form approach. We assume that creditors, due to imperfect information, decide to run on an agent when (i) she has a low level of robustness and (ii) the number of her defaulting counterparties exceeds a certain threshold. In the face of a run, when the agent has to pay back short-term debt, the first line of defense consists of her liquid assets. If these are insufficient, however, the agent may decide to sell under distress some of her long-term assets, in order to pay back the remaining debt. This fire-selling scenario has been modeled in several previous works (e.g., Brunnermeier and Pederson, 2009). Our contribution here consists in combining the fire-selling with the direct loss from the default. Let us emphasize that, fire-selling implies a further loss for the agent, in addition to the initial loss due to the default of the counterparty. This additional repercussion can lead the agent to insolvency, making illiquidity and insolvency two intertwined problems.

In the case of this externality of second type, we find that: (i) for relatively "high" levels of the cross-sectional average robustness, increasing connectivity is always beneficial; (ii) for "low" levels, increasing connectivity does not have any effect on systemic risk, while (iii) for intermediate levels of the average financial robustness, increasing connectivity has first a beneficial and then a detrimental effect. In this case, the reason why diversification can be detrimental is the following. Because of the information incompleteness on the side of the creditors, the probability that a run occurs depends on the absolute number of defaulting counterparties in the credit portfolio of an agent. When there are already a few defaults in the system, then a higher level of diversification implies that more of these defaulting agents can belong to the credit portfolio of the agent. If, in addition, the agent is fragile, there are more chances that the run is triggered.

In summary, in our model diversification is neither always good nor bad. It can have ambiguous effects, and in presence of loss amplification it typically does. The precise outcome depends crucially on the allocation of assets and liabilities across agents and the structure of their mutual exposures. In comparison with the results on diversification found in (Battiston et al., 2009), it should be noticed that while, there, a detrimental effect of diversification results from the dynamics of the network-based financial accelerator - outside the default cascade, here we propose an entirely alternative mechanism which occurs within the cascade.

The paper is organized as follows: Section 2 introduces the model. We define first the structure of the agents' balance sheet. Then, we describe the chain of events triggered by the propagation of losses caused by counterparties' defaults. We derive the dynamics under the two types of external effect in Sections 2.1 and 2.2. In Section 3, we report and discuss the results. Section 3.3 concludes.

\section{The model}

We consider a set of $n$ financial institutions ("banks") connected in a network of borrowing/lending relationships (credit network for short) with each other. As a first approximation, one can think of the credit network as the network of interbank loans. These institutions are also active on "financial markets", i.e. they trade financial obligations with agents outside the network itself. For instance, they can collect deposits, which are liabilities of the banks and assets of agents outside the credit network, i.e. households.
Table 1

Balance-sheet composition. The components of both assets and liabilities are classified in terms of maturity and in terms of whether they generate a financial exposure to some other agents within the financial network. $\mathrm{hh}=$ households, $\mathrm{b}=\mathrm{banks}$.

\begin{tabular}{llll}
\hline Maturity & Nature & Assets & Liabilities \\
\hline Short-term & Network & $A_{i}^{S N}$ (credit to banks) & $L^{S N}$ (debt to banks) \\
Short-term & No Net. & $A_{i}^{S C}$ (cash) & $D$ (hh deposits) \\
Long-term & Network & $A_{i}^{L N}$ (OTC credit) & $L^{L N}$ (OTC loans, b-held bonds) \\
Long-term & No Net. & $A_{i}^{L M}$ (mortgages) & $L^{L H}$ (hh-held bonds) \\
\hline
\end{tabular}

Each institution is represented by a balance sheet. In a sense, we will provide a model of balance sheet dynamics in the following. A similar approach to modeling financial institutions can be found in Eisenberg and Noe (2001) and Shin (2008).

We classify balance sheet items along two dimensions. The first dimension is the maturity (short-term or liquid vs. long-term or illiquid assets/liabilities - "A/L" herefter). The second dimension is the relation (or lack thereof) of the $a / 1$ to the credit network. Some of the a/l, in fact, represent a credit interlinkage as they create a financial exposure to some other agents in the network. Some other a/l, on the other hand, do not represent a credit interlinkage because they are issued and purchased in financial markets outside the credit network.

On the assets' side of the balance sheet of bank $i$, "short-term" assets $A_{i}^{S}$ are liquid, i.e. they can be promptly sold on the market, while $A_{i}^{L}$ are long term illiquid assets, i.e. assets which can be liquidated only at the cost of a non negligible loss of market value. $A_{i}^{k N}, k=S, L$ are assets that represent liabilities of some other agents in the network (for instance interbank loans). There are also two types of assets that are traded on financial markets, i.e. they do not have another financial institution as a counterparty: $A_{i}^{S C}$ can be assimilated to cash available to financial institutions (e.g. bank reserves); $A_{i}^{L M}$ are long term assets such as mortgages or long term bonds.

Similarly, on the liabilities' side, $L_{i}^{S}$ represents short-term debt while $L_{i}^{L}$ is long term debt. $L_{i}^{k N}, k=S, L$ are agent $i$ 's liabilities that represent assets for some other agents in the network: $L_{i}^{S N}$ can be thought of as loans obtained on the interbank market, while $L_{i}^{L N}$ are loans negotiated over-the-counter with other agents. The agent's liabilities towards households are represented by deposits, $D_{i}$, which are short-term in the sense that they are subject to potential withdrawal at any time. Bonds issued by the bank and held by households $L_{i}^{L H}$ are long term because they imply an obligation of reimbursement only at maturity on a time scale longer than the "short term". Table 1 summarizes the composition of the balance sheet.

The difference between total assets $A_{i}$ and total liabilities $L_{i}$ is net worth or the equity base. In the following we will focus on the financial ratio:

$\eta_{i}=\frac{A_{i}^{S C}+A_{i}^{L M}+A_{i}^{S N}+A_{i}^{L N}-L_{i}^{S N}-D_{i}-L_{i}^{L N}-L_{i}^{L H}}{A_{i}^{L N}}=\frac{A_{i}-L_{i}}{A_{i}^{L N}}$,

which is the ratio of equity over the long-term network-related component of assets. This "equity ratio" is an indicator of financial "robustness".

The exercise we carry out in the paper consists first in deriving a law of motion for the financial ratio, in presence of defaults of counterparties. We assume to start from a given initial allocation of assets and liabilities across agents and thus with a given distribution of financial robustness across agents. We also assume some initial defaults. These initial defaults may or not trigger other defaults. In any case, we compute recursively the effect of these defaults on the balance sheets of the counterparties and the counterparties of the counterparties, downstream along all paths in the 

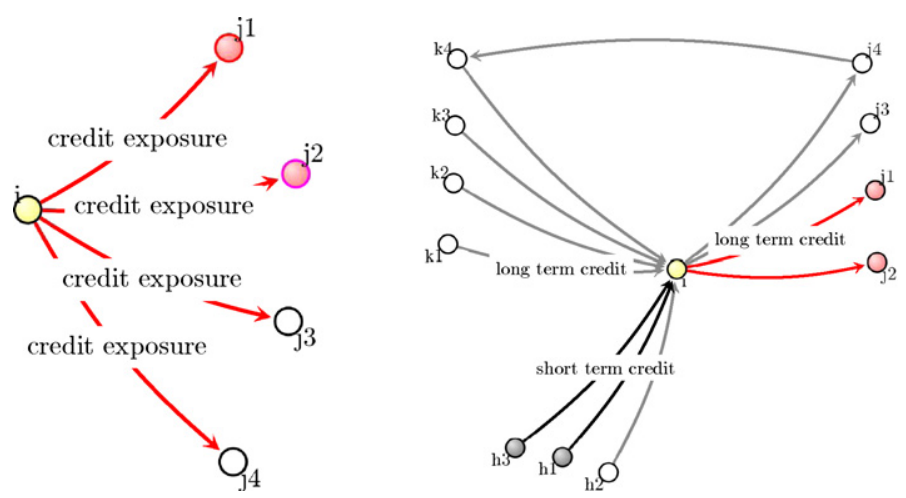

Fig. 1. Representation of the credit relations between agent $i$ and its obligors in the simplest case (left). Another scenario: agent $i$ has obligations to both long-term creditors $\left(k_{1}-k_{4}\right)$ and short-term creditors $\left(h_{1}-h_{3}\right)$, while it has long-term claims on some obligors $\left(j_{1}-\mathrm{J}_{4}\right)$ ) (right).

network. At the end of this cascading process there is a number of default accumulated. We will study how the cascade dynamics is affected by the distribution of initial level of robustness across agents and on the network structure. At the end of one cascading process all agents are replaced by new agents. This is a sort of stress test for the whole financial network, in which the shock is represented by the initial default, and the response is given by the final number of defaults.

In terms of informational set, agents are assumed to know which agents have defaulted, but they do not know the exposures of their counterparties to other agents. Only in the extreme case of fully connected network, connections are trivially known to everyone. Moreover, agents do not know with certainty the level of robustness of their counterparties. Therefore, they cannot anticipate whether there will be a large default cascade or not. Fig. 1 shows a visual illustration of the situations leading to two different types of external effects. In the first situation (left), the default of some counterparties affect agent $i$, but there is no further repercussions. In the second situation (right), the presence of short-term lenders creates the potential for further repercussions on $i$ herself, as it will be clear in the following section.

\subsection{External effect of the first type}

Suppose that counterparty $j$ of agent $i$ defaults on her long-term obligations at time $t$. This affects $A_{i}^{L N}(t)$, i.e. the network-related long-term component of the balance sheet in the same period, which will be reduced by a fraction $a$ of the nominal value of the obligation of agent $j$ to $i, A_{i j}^{L N}(t)$ :

$A_{i}^{L N}(t)=A_{i}^{L N}(t-1)-a A_{i j}^{L N} \chi_{j}^{(I)}(t)$,

where $\chi_{j}^{(I)}(t)$ indicates if agent $j$ defaults at time $t$. The parameter $a$ measures the fraction of funds that agent $i$ is assumed to loose, in the short-term, when the counterparty $j$ defaults. For sake of simplicity, here, this fraction $\alpha$ is assumed to be the same across agents, but it could be made heterogeneous. With $a=0$, banks do not loose anything, which implies that default do not have any externality on the counterparties.

It is not unfrequent that defaults propagate on the time scale of days or weeks, as it has been the case during the various episodes observed during the fall of 2008. Such short time horizon makes reasonable the following assumptions. First, agents are not able to modify their exposures to other agents in reaction to the defaults occurring in the financial network. Secondly, agents are not able to recover, within the duration of the cascade, most of the proceeds from the liquidation of the assets of the defaulted counterparties, so that $a \approx 1 .{ }^{1}$ We will discuss the case $a<1$ at the end of Section 3.2.

Moreover, in the scenario we are looking at, within the duration of a default cascade, agent $i$ 's assets at time $t$ are affected by the bankruptcy of agent $j$ independently of the period in which her bankruptcy has occurred. It is then convenient to rewrite the equation above as follows:

$A_{i}^{L N}(t)=A_{i}^{L N}(0)-a A_{i j}^{L N} \chi_{j}(t)$,

where $A_{i}^{L N}(0)$ represents the "initial value" of network-related longterm assets (in the following the initial condition will be denoted as the beginning of a cascade of defaults), and $\chi_{j}(t)$ indicates if agent $j$ has defaulted at time $t$ or in any period before $t$.

Considering the nexus of credit interlinkages, the total loss to agent $i$ due to non-performing loans ("bad debt") can be indicated by $\sum_{j} A_{i j}^{L N} \chi_{j}(t)$. Agent $i$ becomes insolvent when net worth becomes negative:

$A_{i}^{S C}+A_{i}^{L M}+A_{i}^{S N}+A_{i}^{L N}-a \sum_{j} A_{i j}^{L N} \chi_{j}(t)-L_{i}^{S N}-D_{i}-L_{i}^{L N}-L_{i}^{L H}<0$,

where to simplify the notation, we dropped the indication of the time 0 . Let us denote the relative exposure of $i$ to $j$ in terms of long term liabilities as follows:

$W_{i j}=\frac{A_{i j}^{L N}}{A_{i}^{L N}}$.

From this definition follows that the matrix $W$ of relative exposures is non-negative and row-stochastic. Dividing both sides of Eq. (4) by $A_{i}^{L N}$ and recalling the definition of the equity ratio above we conclude that solvency requires the following quantity to be positive

$\eta_{i}(t)=\eta_{i}(0)-a \sum_{j} W_{i j} \chi_{j}(t)$

$\eta_{i}(0)$ represents the initial financial robustness of $i$. The term $\sum_{j} W_{i j} \chi_{j}(t)$ accounts for all relative losses (due to the default of some of her counterparties) experienced by agent $i$ since the beginning of the cascade. ${ }^{2}$ Notice that this measure of financial robustness is an inverse measure of aggregate asset risk. Thus, the same argumentation of the paper can be recast in terms of asset risk.

Let us now assume that each agent $i$ with $k_{i}$ counterparties, has roughly comparable exposure to them. Then, it is $W_{i j}=1 / k_{i}$ (uniform risk sharing). Let us indicate with $k_{f}$ the number of defaulting partners of agent $i$. Hence $\sum_{j} W_{i j} \chi_{j}(t)=k_{f i} / k_{i}$ is the fraction of defaulting counterparties, which measures the relative impact of defaults on financial robustness. In the case of uniform risk sharing, therefore, the law of motion of the equity ratio above can be written as follows:

$\eta_{i}(t)=\eta_{i}(0)-a \frac{k_{f i}}{k_{i}}$

Eq. (7) implies that the fraction of defaulting counterparties is the main determinants of the default cascade (as also found in (Gai and Kapadia, 2010a)). However, it may not be obvious at a first thought that the same level of relative loss $k_{f i} / k_{i}$, can occur with

\footnotetext{
1 For instance one can assume that defaulted banks' assets are distributed to depositors first and to other creditors next, as in (Iori et al., 2006).

2 Notice that the dynamics of insolvency is completely independent of introducing $\eta$ and normalizing as we have done. The procedure simply allows to rewrite the dynamics in a convenient way.
} 
very differing probability, depending (1) on the values of $k_{f}, k_{i}$ and the probability $p$ of individual defaults, and (2) on the presence (or lack thereof) of correlations. Let us illustrate this statement with some examples.

If defaults are uncorrelated, the probability $P\left\{k_{f i}, k_{i}\right\}$ of occurrence of $k_{f i}$ defaults among $k_{i}$ partners follows a binomial

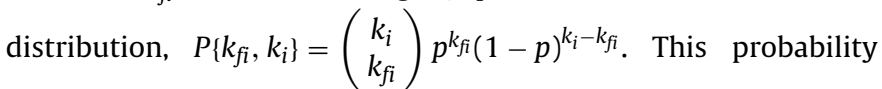
decreases sharply with $k_{f i}$, for $k_{f i}>k_{i} p$, for given $k_{i}$ and $p$. In particular, for a given value of $p$ and $k_{f i} / k_{i}$, the larger is $k_{i}$, the less probable it is to observe $k_{f}$ simultaneous defaults. For instance, consider $p=0.1$ and $k_{f i} / k_{i}=0.2$. The probability to have 2 defaults out of 10 counterparties $(P\{2,10\} \sim 0.2)$ is much larger than the probability to have 20 defaults out of $100(P\{20,100\} \sim 0.001)$. Thus, when defaults are not correlated, the event consisting of several simultaneous defaults among the counterparties of an agent with a large portfolio is quite rare.

If instead defaults are correlated, the probability of simultaneous defaults is not binomial anymore. In contrast to the uncorrelated case, it may be more likely to observe many simultaneous defaults than only few ones. Unfortunately, there is no simple way to describe mathematically such probability, as it depends on the structure of the correlation (Frey and McNeil, 2003).

Notice that, even if the average individual default probability is known (or can be estimated from the frequency of defaults in the whole population), the probability of joint defaults remains unknown if the correlation is unknown. But the correlation of defaults in a network depends itself on the default probability and the network structure. For our purposes, it suffices the assumption that either defaults are uncorrelated or they are completely correlated, as it will be clear in Section 3.

In the dynamics of $\eta_{i}$ presented above, the shock caused by the default of one agent is uniformly spread among the creditors. This is the external effect of type 1 . The external effect of defaults is, in a sense, conservative because it is divided across the agents who bear some financial exposure to the defaulting partner but it does not get amplified during its propagation. In a more realistic setting, in which the lender can recover at least part of the interbank claim on defaulted agents, the external effect of type 1 would be mitigated. In the present framework, for sake of simplicity and based on the assumption (see earlier) that the legal settlement of the bankruptcy takes much longer than the unraveling of the cascade, we rule out repossession of the assets of the defaulted agents on the part of the lender.

\subsection{External effect of the second type}

In this section, we focus on another type of external effects in which the losses due a counterparty default can cause additional losses to the agent. Losses are - in a sense - amplified along each connection. In this paper, we investigate how this type of effect can arise in a specific scenario ${ }^{3}$ in which a liquidity run and the consequent fire-selling on the asset side generates a further repercussion to the agent, in addition to the first direct loss due the counterparty default. In a sense, the external effect of the second type combines the externality of the first type with the fire-selling scenario already well investigated in the literature (see e.g., Brunnermeier and Pederson, 2009). In the following, we first discuss how illiquidity originating from the default of one or more counterparties of an agent can lead to her insolvency, due to the possibility of a run of its

\footnotetext{
${ }^{3}$ Other scenarios, e.g., involving credit derivatives would be plausible candidates, but they are not investigated here.
}

short-term lenders. Notice that the run is not modelled explicitly. We assume that the run occurs or not depending on the level of robustness of the agent and on the number of her counterparties in default. Based on such assumption, we derive a law of motion for robustness that incorporates the external effect of second type.

An agent $i$ facing the request to repay a part of her liabilities denote such amount as $\Delta L_{i}^{S N}$ - becomes illiquid in case her liquid assets are not sufficient to cover for the payment:

$A_{i}^{S N}+A_{i}^{S C}-\Delta L^{S N}<0$.

An agent can well be illiquid even if she remains solvent - i.e. even if net worth remains positive - (see Eq. (4)).

In terms of informational set, we have in mind the situation in which short-term creditors do not know with certainty the level of robustness of the counterparties of the agent. In the presence of this information imperfection, the default of one or more of the counterparties of $i$, can trigger the illiquidity of $i$, even if she can absorb the shock due to the defaults and remain solvent. This happens in two stages.

Firstly, the default of some counterparties is a shock that reduces the asset side of $i$ and thus, in presence of uncertainty on the future, increases the chances of default of $i$. More specifically, recall that, as discussed earlier, the probability of occurrence of the relative loss due to defaults, $k_{f i} / k_{i}$, depends in non-trivial way on the values of $k_{f}$, $k_{i}$, the probability $p$ of individual defaults, and on the presence of correlation. Thus, when short-term creditors of agent $i$ observe a number of simultaneous defaults among her counterparties, they face uncertainty along several dimensions: Has the probability of default increased? Were there interdependencies among the counterparties who defaulted? Does this mean that the remaining counterparties are also likely to fail shortly after? Finally, is there something systematically wrong in the way the agent has chosen her counterparties?

In other words, imperfect information on the financial health of the counterparties of agent $i$ and the structure of the correlations among their possible defaults, implies that short-term lenders cannot exclude that the probability of default of $i$ has increased, even if at the moment she is still solvent. It follows - and this the second step - that it is rational for the short-term lenders of $i$ to consider the option of refusing to roll-over debt to $i$. This is even more so, given that, at the same time, all other short-term lenders may also end up deciding not to roll-over, i.e., that there could be a run on agent $i$.

To continue with the sketch of the situation we imagine here, let us assume that, as a result of the defaults among $i$ 's counterparties, some of $i$ 's lenders refuse to roll-over their short-term loans and agent $i$ will have to pay back short-term debt. She will first try to satisfy this need using her liquid assets $A f$. If these are not sufficient, i.e. $\left|\Delta L_{i}^{S N}\right|>A_{i}^{S}$, then $i$ may decide to sell some of her long-term assets. For the sake of simplicity, in the following we assume that the first line of assets sold under distress consists of securitized mortgages. If the market of such type of securities is liquid the agent is able to re-balance her liquidity position without any loss. However, if the market is not very liquid agent $i$ may be forced to a fire-selling, i.e. to sell below market price. Then, the nominal value of assets to be sold exceeds, in absolute value, the value of liabilities to be repaid:

$\left|\Delta A_{i}^{L M}\right|=q\left(\left|\Delta L_{i}^{S N}\right|-A_{i}^{S}\right)$,

where $q=p^{\text {(market) }} / p^{\text {(fire) }} \geq 1$ is the ratio of the market price over the selling price. ${ }^{4}$ Whether and when creditors will eventually run

\footnotetext{
4 Incidentally, notice that in Gai and Kapadia (2010b), as a result of counterparties defaults and short-term lender refuse to roll-over, agents decide to hoard liquidity
} 
- i.e. refuse to roll over to $i$ - is an issue that has been extensively investigated in the vast literature on bank runs. Most works find that investors run when the shock hitting the bank is relatively large and the bank is already relatively fragile (Rochet and Vives, 2004). In line with this literature and motivated by the previous discussion, we assume (without modeling the coordination game among creditors) that there is a run of all the creditors if the number of defaults is larger than a certain threshold that increases with the robustness of the agent,

$k_{f i}>\gamma \eta_{i}(0)$,

where $\gamma$ is a scale factor, with $0 \leq \gamma \leq 1$. In other words, there is a run when several counterparties default and the agent has already low robustness.

Notice that when the run occurs, agent $i$ has to repay an amount corresponding to the total aggregate short-term loan she received, i.e. $\left|\Delta L_{i}^{S N}\right|>L_{i}^{S N}$

$L_{i}^{S N}$, since all the creditors run. This is done by reducing $A L_{i}^{M}$ according to Eq. (9). As a result, in case of a run after the defaults, the equity of $i$ decreases as follows:

$$
\begin{aligned}
A_{i}(t)-L_{i}(t) & =A_{i}-L_{i}-a \sum_{j} A_{i j}^{L N} \chi_{j}(t)-A_{i}^{S}-\Delta A_{i}^{L M}+\Delta L_{i}^{S N} \\
& =A_{i}-L_{i}-a k_{f i} A_{i j 0}^{L N}-A_{i}^{S}-q\left(\Delta L_{i}^{S N}-A_{i}^{S}\right)+\Delta L_{i}^{S N} \\
& =A_{i}-L_{i}-a k_{f i} A_{i j 0}^{L N}-(q-1)\left(L_{i}^{S N}-A_{i}^{S}\right) .
\end{aligned}
$$

After normalizing by $A^{(L N)}$, we finally obtain the following dynamics:

$\eta_{i}(t)\left\{\begin{array}{l}\eta_{i}(0)-a \frac{k_{f i}}{k_{i}}-b_{i} \text { if } \eta_{i}(0)<\gamma k_{f i} \\ \eta_{i}(0)-a \frac{k_{f i}}{k_{i}} \text { otherwise }\end{array}\right.$,

where the parameter

$b_{i}=(q-1) \frac{L_{i}^{S N}-A_{i}^{S}}{A_{i}^{L N}}$,

measures the impact on agent $i$ of the cost of the run $(q-1)$ $L_{i}^{S N}-A_{i}^{S}$, relative to the long-term network related assets. Notice that, in addition to the shock due to $i$ 's counterparty defaults, in case of run, agent $i$, faces now a further decrease of equity. This amplification of the initial shock characterizes what we call external effect of the second type. The idea that a liquidity run can trigger a fire-selling is well-known. Our contribution here is to cast this within the framework of the default cascade and show that its effect is to amplify the initial losses. Notice also, that if we rule out runs for all agents, either because they do not make use of short-term credit, or because the threshold is never reached $(\gamma=0)$, this is equivalent to set $b_{i}=0$, so that we recover the dynamics with external effects of the first type (Eq. (6)).

For the sake of simplicity, in the following we focus on the case in which the parameter $b_{i}$ is homogenous across agents, $b_{i}=b$ for all $i$.

\section{Default cascades}

The initial default of one or more agents in the credit network triggers the default of other agents, i.e. a cascade or avalanche of

by withdrawing short-term lending from other agents in the network. Here we do not focus on the liquidity evaporation issue, but on the propagation of insolvency. defaults. The development of a cascade in the model is generated applying recursively the dynamics of Eq. (11). The cascading process is deterministic and terminates after a finite number of steps. ${ }^{5}$ In the final state of the cascade, a certain fraction $s$ of the agents has defaulted. This is the size of the cascade, which, in our framework, captures the magnitude of the systemic risk to which the credit network is exposed. In the following, we investigate how to determine the size of the cascade and the role played by diversification for financial stability.

The fraction of defaults at the end of the cascade (i.e., the size of the cascade) can be computed as the stable fix point of a recursive equation for the cumulative fraction of failures. We will derive this equation first in the presence of externalities of the first type ${ }^{6}$ and then in the general case of external effects of the second type.

\subsection{External effects of the first type}

We first focus on the dynamics described in Section 2.1 by Eq. (6). It is instructive to illustrate a simplified version of the computation method. Suppose the network is a regular graph, i.e. each agent has the same number of connections $k$. The fraction $s(t+1)$ of agents who will default in the future time step of the dynamics represented by Eq. (6) is simply the fraction of agents who happen to go below the default threshold in the current time step. In a large system, such fraction approximates the probability that the robustness of a randomly chosen agent is below the threshold. Moreover, in a mean-field approximation, we can replace the expected fraction of defaults among the counterparties of each agent with the current fraction of defaults in the population. We can thus write

$s(t+1)=P\left\{\eta_{i}-\frac{k_{f i}}{k}<0\right\} \approx P\left\{\eta_{i}<s(t)\right\}$

Assuming that agents who default are not replaced or refinanced during the cascade, then the cascade size is at least the initial fraction of defaults $s(1)$. We, thus, obtain the following equilibrium condition:

$s=F(s)=\max \{s(1), \Phi(s)\}$,

where $\Phi(x)=\int_{-\infty}^{x} p(\eta) d \eta$ is the cumulative distribution of robustness up to the value $x$. This yields a recursive equation in $s$ whose solution is the size of the cascade. One can improve the computation using a better estimation of expected number of defaults among the counterparties of a given agent. However, the procedure is conceptually the same.

The outcome of the cascade depends of course on the initial distribution of robustness across agents. If agents have low robustness, the cascade tends to be larger because the default of some agents is more likely to cause the default of other agents. In the following, we will assume that the initial distribution of robustness when the cascade starts can be approximated by a Gaussian with mean $m$ and standard deviation $\sigma_{\eta}$. The values of mean and standard deviation have an impact on the shape of the function in Eq. (14): a decrease of robustness shifts the function $F(s)$ to the right, while a decrease of $\sigma_{\eta}$ makes the slope steeper. In Fig. 2 (left and right), the function $F(s)$ is plotted for some values of $m$ and $\sigma_{\eta}$ and two examples of fixed points are illustrated.

Notice that if the initial robustness is distributed across agents according to a Gaussian distribution, there is always a positive probability that some agents have a value of robustness below 0 ,

\footnotetext{
5 This number is smaller than the number of agents since agents are not re-started if failed during the cascade (Kleinberg, 2007).

${ }^{6}$ Notice that this method differs from the graph generating function approach used in (Gai and Kapadia, 2010a), which builds on (Callaway et al., 2000).
} 

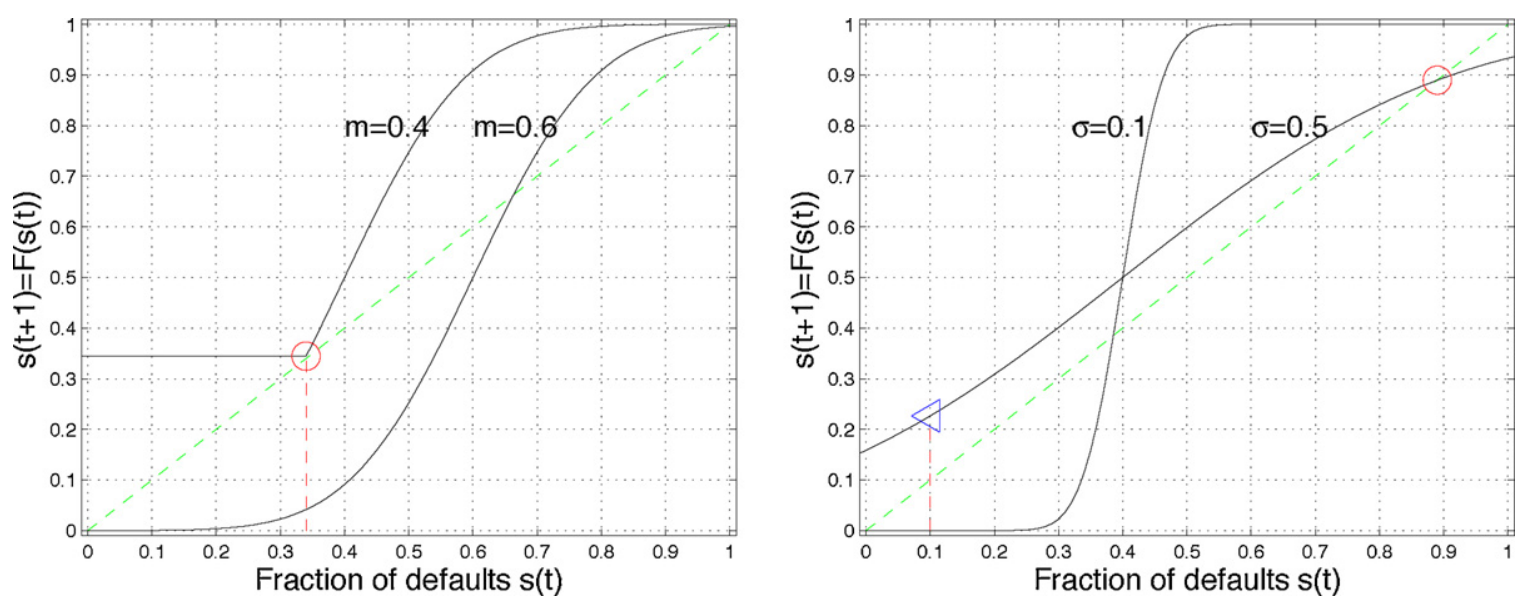

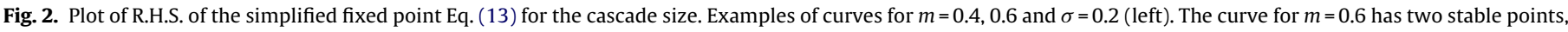

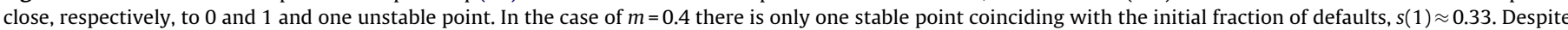

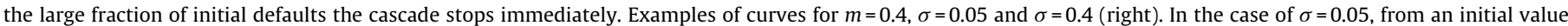
$s(1) \approx 0.1$, the fraction of defaults ends up close to a complete default, $s \approx 0.9$.

because the support of the Gaussian stretches to the whole real line. ${ }^{7}$ In general, there is an expected number of initial defaults that depends simply on the mean and standard deviation of the distribution. We denote these initial defaults as endogenous.

However, in line with the spirit of a stress testing exercise, we are interested in investigating the response of the financial network, given a certain distribution of robustness, to shocks of varying intensity. Therefore, in addition to the endogenous defaults, we assume that, at time 0 , a fraction $y_{0}$ of randomly chosen agents default. By varying $y_{0}$, we vary the intensity of the shock hitting the financial network at the beginning of the cascading process. We denote these defaults as exogenous.

Notice that in this modelling framework one can also investigate the effect of systematic shocks hitting all agents in the same way. Indeed, one can study, as we do in the following, the outcome of the cascade for varying levels of the average robustness across agents. Shifting the mean of the distribution down, say from $m_{1}$ to $m_{1}-\in$ is equivalent to assuming that a systematic shock $\in$ has hit all agents before the dynamics starts.

The following proposition characterizes the size of the cascade.

Proposition 1. Consider the process of Eq. (6). Assume the network of firms is a regular random graph with degree $k$. Assume also the initial probability distribution of robustness is Gaussian ${ }^{8}$ with mean $m$ and variance $\eta^{2}=\sigma^{2} / k: p(\eta(t=0)) \sim \operatorname{Gauss}\left(m, \sigma_{\eta}\right)$. Let $\Phi(x)=\int_{-\infty}^{x} p(\eta) d \eta$ denote the cumulative probability distribution of $\eta$ up the value $x$, and $s_{0}=\Phi(0)$ denote the fraction of firms whose robustness is below zero at $t=0$. Moreover, assume that at time $t=0$ there is a fraction $y_{0}$ of exogenous defaults. Then:

1. The fraction $s$ of failures at the end of the cascade process is the solution of

$$
s=\max \left\{s_{0}+y_{0}, F\left(s, m, \sigma_{\eta}\right)\right\}
$$

\footnotetext{
7 It would be interesting, to know the empirical distribution of the variant of equity ratio, $\eta_{i}$ that we have introduce in this paper. However, differently from the case of the usual equity ratio, there are no readymade statistics and one would need to look at the balance sheets of the institutions in order to estimate the amount of assets that have a network valence.

${ }^{8}$ A similar computation could be carried out for other probability distributions of robustness $p(\eta)$
}

$$
F=\sum_{k_{f=1}}^{k}\left(\begin{array}{c}
k \\
k_{f}
\end{array}\right) s^{k_{f}}(1-s)^{k-k_{f}} \Phi\left(\frac{a k_{f}}{k}\right)
$$

\section{A stable fixed point always exists.}

Notice that Eq. (15) provides an analytical expression for the cascade size. ${ }^{9}$ The result holds also for random graphs in the limit of small degree variance. It is possible to extend the result to the case of heterogenous networks with a given degree distribution $p(k)$. In the expression of $F$, one needs to also sum over varying level of the connectivity degree $k$, and to weight each term by the corresponding value of probability $p(k)$. However, in so doing we would introduce additional dimensions to the parameter space. This case will be investigated in future work.

In this paper we want to focus on the effect of the average level $k$ of the diversification and how it affects the cascade size $s$, depending on the values of the parameters $\sigma, m$ and the exogenous shock $y_{0}$. We find, that typically, the cascade size is either $s_{0}+y_{0}$ itself (i.e., no new defaults are induced by the initial ones), or a full cascade of the whole financial system is triggered. In particular, there are different regimes, in which individual risk diversification may have either a beneficial (stabilizing) macroeconomic role, or a detrimental one, or no role at all. Further work should aim at deriving some general results on the exact transition boundaries between these regimes in the space of the parameters $\sigma, m$ and $y_{0}$. However, even if these general results are not yet available, the proposition above allows to prove (simply by existence) a few interesting results and to draw some relevant implications for financial stability. Findings are illustrated in scenarios, related to specific regions of the parameter space. To help the reader getting a general picture, the detailed description of each finding is preceded by a concise but simplified statement. All results concern the default cascade size depending on the following parameters: the standard deviation $\sigma$ and mean $m$ of the robustness distribution, the size of the exogenous shock $y_{0}$ and the connectivity degree $k$ of the agents in the network.

In order to better understand the implications of the findings, let us recall the meaning of the parameters and their range of variation.

\footnotetext{
${ }^{9}$ As for all trascendental equations, i.e., involving non-polynomial functions, the solution has to be computed numerically. But this can be done with arbitrary precision. This is not the same as finding the cascade size with simulations.
} 
The average financial robustness $m$, ranges in [01]. A value $m=0$ means no equity. Because the distribution is Gaussian and thus symmetric, $t$ also means that half of the agents have robustness below 0 . Instead, $m=1$ means that equity is as large as long-term network-related assets (see Eq. (1)). Given the range of $m$, values of the standard deviation of robustness $\sigma>0.5$ can be considered as large, while values $\sigma<0.1$ are small. Finally, since $y_{0}$ represents the initial fraction of defaulting agents, values $y_{0}>0.3$ are quite large, while $y_{0}<0.05$ represent typical situations in normal course of the economy.

Scenario 1. A fragile system is prone to systemic default, even if there are no exogenous shocks. Even in the absence of exogenous defaults, $y_{0}=0$, for any value of the standard deviation $\sigma$, the cascade size $s$ tends to 1 for decreasing values of $m$. Fig. 2 shows how the fix point solution of Eq. (13) varies as a function of $m$ and $\sigma$. A decrease in $m$ shifts to the right the function $F$. The slope of $F$ decreases with $\sigma$. However, it is clear that no matter how steep is the slope (i.e., small $\sigma$ ) there is always a value of $m$ small enough so that $F(s)>s$ for all $n \in$ ] 01 [and thus the only stable point is the one closer to $s=1$. The same result applies to Eq. (15). Thus, when the average robustness across agents is low enough, the endogenous defaults trigger a systemic default even in the absence of any exogenous shock. In this case, diversification is irrelevant, as shown in Fig. 3. Indeed the size of the cascade remains constant with $k$ and close to 1 , when the average robustness is low.

Scenario 2. Diversification prevents systemic defaults when the financial condition overall is "not too bad". There exists a range of the parameter values $(\sigma, m) \approx[00.15] \times[0.20 .5], y_{0}<0.1$, where the cascade size $s$ decreases with diversification $k$. Fig. 3 (right) shows that, for a given $m$, $s$ eventually drops to small values as $k$ increases.

Thus, when financial robustness is not very different across agents (small variance of robustness) and the exogenous shock is not large, then diversification makes the system more resilient to systemic defaults. More precisely, with a larger diversification, the system remains stable even at lower values of average robustness. This result is in line with the pro-connectivity view mentioned in the Introduction. However, results are not always in this direction.

Scenario 3. Diversification may in some cases lead to systemic defaults. There exists a range of values, e.g., $(\sigma, m) \approx[0.41] \times[0.20 .8]$, where the cascade size $s$ increases with diversification $k$, as shown in Fig. 4 (left). This is a quite counterintuitive result which requires a more detailed explanation.

First of all, it is not always necessary a big loss to cause the default of an agent. If her robustness is low, i.e. her equity is small relative to her assets, even a relatively small loss may be sufficient to push the agent beyond the threshold of default. This depends on the structure of assets and liabilities, as defined by the solvency condition in Eq. (4). What happens then, when an agent suffers a loss bigger than the critical one, i.e. the loss which would be exactly sufficient to make her default? The excess loss - i.e. the difference between the actual and the critical loss - does not have any additional effect on the agent and her creditors. Then consider the case in which initial robustness is heterogeneous and many agents are fragile. With a low level of diversification, the initial default of an agent causes a big loss to counterparties that are already fragile. These counterparty fail, but many more could have failed, would the loss been shared more broadly. So the momentum of the initial loss is dampened on the way.

Instead, when the level of diversification is higher, then every default adds only a little loss to each counterparty. Thus, when the accumulated losses eventually exceed the solvency threshold, they do so by little margin, so that "no loss is lost", so to say. In other words, the momentum of the initial defaults is not dampened on the way. This implies that there can be situations in which the initial endogenous and exogenous defaults are capable of triggering a systemic default when the diversification is high, while the cascade stops on the way when the diversification is low.

This feature obviously depends on the way the propagation of distress has been defined in the model, which includes a sort of limited liability of agents. Still, the result raises an interesting issue, in particular in connection to how the losses associated to defaults are socialized.

Scenario 4. Diversification has no effect when the system is fragile, relatively to the exogenous shock. There exists a range of parameter values, i.e. $(\sigma, m) \approx[00.4] \times[00.2]$, with $y_{0}>0.1$ where $s$ does not change with $k$ (see Fig. 4, right). Thus, if robustness is not very heterogeneous and the average is low, then varying the level of diversification has no effect. The systemic default occurs anyway. There is no gain in distributing losses more widely because the exogenous shock is already big enough to knock down everybody. There is also no gain in concentrating the losses among fewer counterparties because the most robust agents are not much more robust than the weak ones.

\subsection{External effects of the second type}

In this section, we apply the procedure already described in the previous section to the dynamics defined in Eq. (11). We also make an additional assumption concerning the heterogeneity of robustness across agents. One may argue that, if agents have a high level of diversification in their financial exposure, then this affects not only the loss they face as a result of the default of a counterparty but also the variations in the initial robustness from an agent to another. To put it straight, imagine each agent diversifies its exposure, with equal weights, on the entire set of other agents. Then, the individual robustness may still vary, since it depends on the choice of the level of short-term vs. long-term assets and liabilities. However, differences in robustness between any two agents should decrease because all agents have almost the same portfolio of $A^{L N}$ assets. Notice that if the standard deviation $u$ of the robustness decreases, then, as long as the average $m$ is positive, the fraction of endogenous defaults, i.e. the agents that are already initially below 0 , decreases. This can be expected to make the system more resilient. Thus, in order to verify if the results found in the previous sections still hold when the heterogeneity decreases with the diversification, we assume that the variance of the robustness scales as $\sigma_{\rho}^{2}=\sigma^{2} / k_{i}$. This is a marked decrease in heterogeneity - variance tends to zero for large $k_{i}$ - which corresponds then to quite a conservative scenario. Notice that the assumption is in line with (Battiston et al., 2009), where the financial robustness is the result of an endogenous dynamic process. The size of the cascade under the dynamics including credit runs is characterized in the following proposition.

Proposition 2 (.). Consider the process of Eq. (11). Assume the network of firms is a regular random graph with degree $k$. Assume also the initial probability distribution of robustness is Gaussian ${ }^{10}$ with mean $m$ and variance $\sigma_{\eta}^{2}=\sigma / k: p(\eta(t=0)) \sim \operatorname{Gauss}\left(m, \sigma_{\eta}\right)$. Let $\Phi(x)=\int_{-\infty}^{x} p(\eta) d \eta$ denote the cumulative probability distribution of $\eta$ up the value $x$, and $s_{0}=\Phi(0)$ denote the expected fraction of firms whose robustness is below zero at $t=0$ (endogenous defaults). In addition, assume that at time $t=0$ there is a fraction $y_{0}$ of exogenous defaults. Then:

\footnotetext{
${ }^{10}$ A similar computation could be carried out for other probability distributions of robustness $p(\eta)$
} 

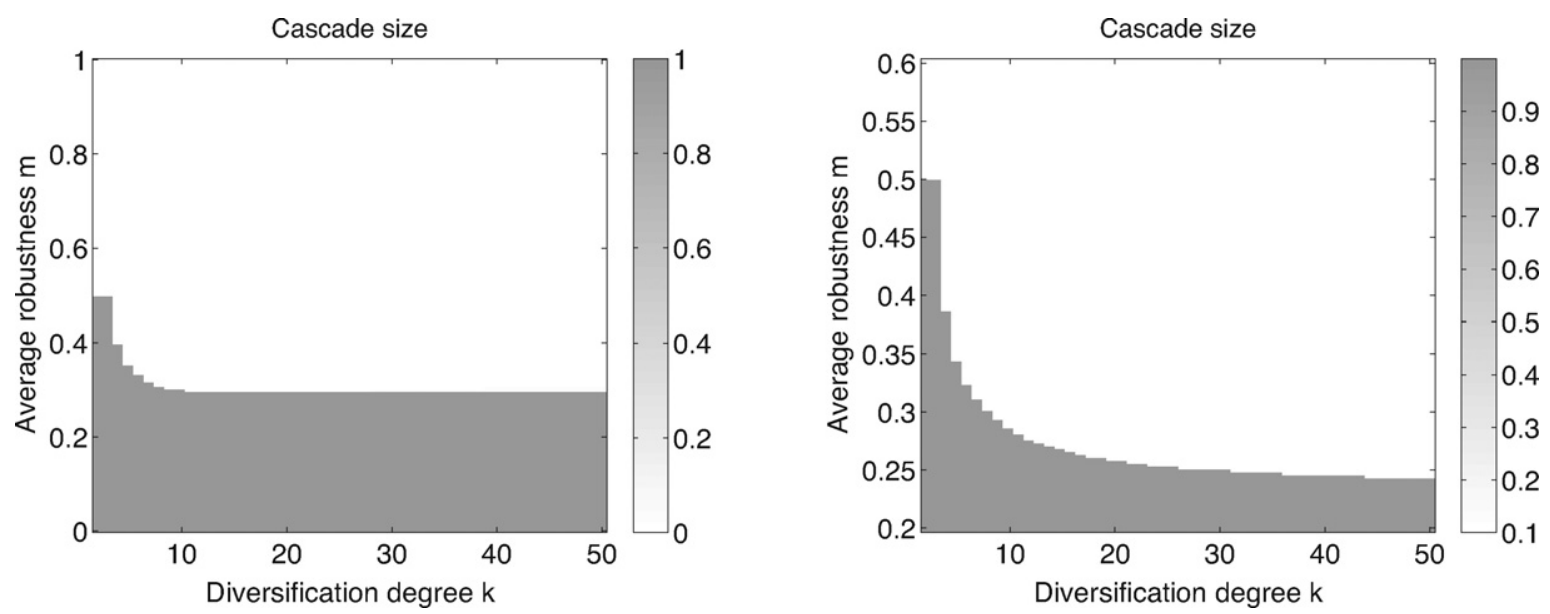

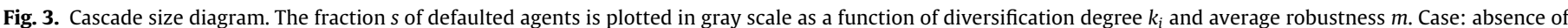
exogenous shock $y_{0}=0$, intermediate variance, $\sigma=0.2$ (left). Case: $y_{0}=0.1, \sigma=0.1$ (right).

1. The fraction s of failures at the end of the cascade process is the solution of the equation

$$
\begin{aligned}
s= & \max \left\{s_{0}+y_{0}, G\left(s, m, \sigma_{\eta}, b \gamma\right)\right\} \\
G= & \sum_{k_{f=1}}^{k}\left(\begin{array}{c}
k \\
k_{f}
\end{array}\right) s^{k_{f}}(1-s)^{k-k_{f}}\left(\Phi\left(\frac{a k_{f}}{k}\right)\left(1-\Phi\left(\gamma k_{f}\right)\right)\right. \\
& \left.+\Phi\left(\frac{a k_{f}}{k}+b\right) \Phi\left(\gamma k_{f}\right)\right)
\end{aligned}
$$

\section{A stable fixed point always exists.}

Again, we study how the level of diversification k impacts on the cascade size $s$. In addition to the parameters $\sigma, \mathrm{m}$ and $y_{0}$ (respectively, standard deviation and mean of robustness, and exogenous shock) already at play in Section 3.1, we have here one more parameter, namely the cost of the credit run, $b$. As before, we observe different regimes for the behavior of the cascade size.

The meaning of the values of the parameters $m, \sigma$ and $y_{0}$ has been explained in the previous section. For the scale factor $\gamma$ a value close to 0 means that short-term lenders are very little sensitive to the defaults among $i$ 's counterparties. For instance if $k_{f}=3$ and gamma $=0.01$, then a run occurs only of agent $i$ has an initial robustness smaller than $\gamma k_{f}=0.03$ (see Eq. (11)).

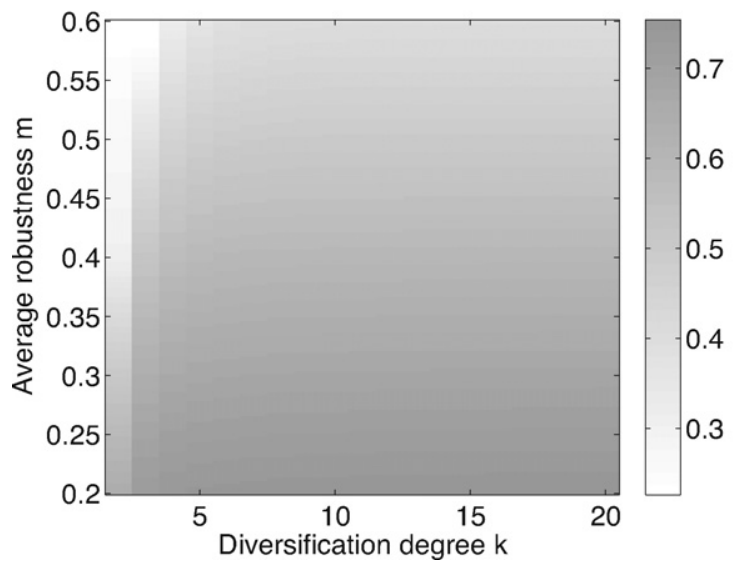

Finally, to give a concrete idea of the meaning to the values of the parameter $b$, consider the following relatively severe scenario: a marginal cost of fire-selling of $50 \%$, i.e., $q-1=0.5$; a small amount of cash and a large ratio of short-term network-related liability to long-term network-related assets, so that, e.g., $L_{i}^{S N}-A_{i}^{S C} / A_{i}^{L N}=0.8$. This yields a value $b=0.4$ for the impact of the cost of the run (see Eq. (12)). Thus, values $b<0.1$ correspond to mild cost of runs while values $b>0.3$ correspond to significant cost of runs.

Scenario 5. In the absence of runs and large exogenous shocks, diversification prevents systemic defaults. There exists a range of parameter values $\sigma \sim[00.3], y_{0}<0.1$ where cascade size $s$ decreases with diversification $k$ for a given average robustness $m \in[0.11]$ (see Fig. 5, left). Thus, when the exogenous shock is not very large, then diversification makes the system more resilient to systemic defaults. More precisely, with a larger diversification, the system remains stable even at lower values of average robustness. In particular, the result suggests that, as long as robustness is positive, there is always a level of diversification (provided the number of agents is also large enough) so that systemic defaults disappear. Again, in this parameter range the behaviour supports the proconnectivity view.

Scenario 6. In the presence of runs, diversification has an ambiguous effect. There exists a range of parameter values, e.g., $(\sigma, m) \approx[00.5] \times[0.10 .5], b>0.2, \gamma>0.02$, where the cascade size $s$ first decreases and then increases with diversification $k$ (see Fig. 5 ,

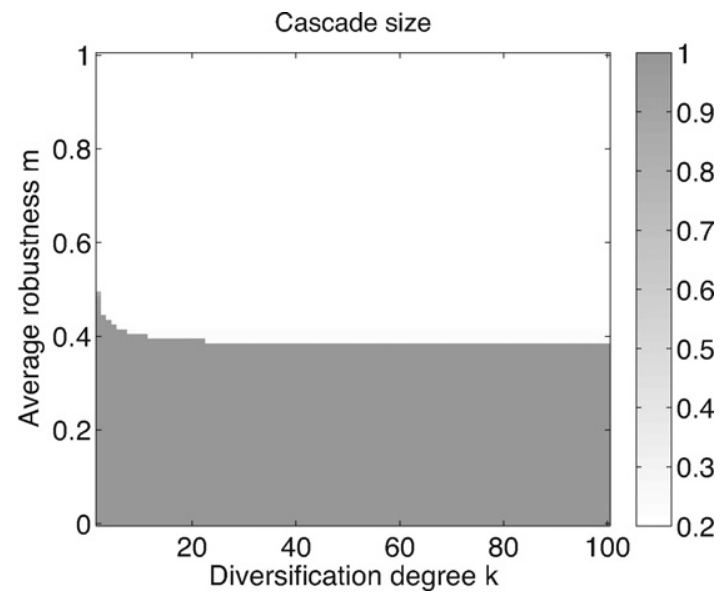

Fig. 4. Cascade size diagram (see Fig. 3). Case: $y_{0}=0, \sigma=0.8$ (left). Case: $y_{0}=0.2, \sigma=0.2$ (right). 

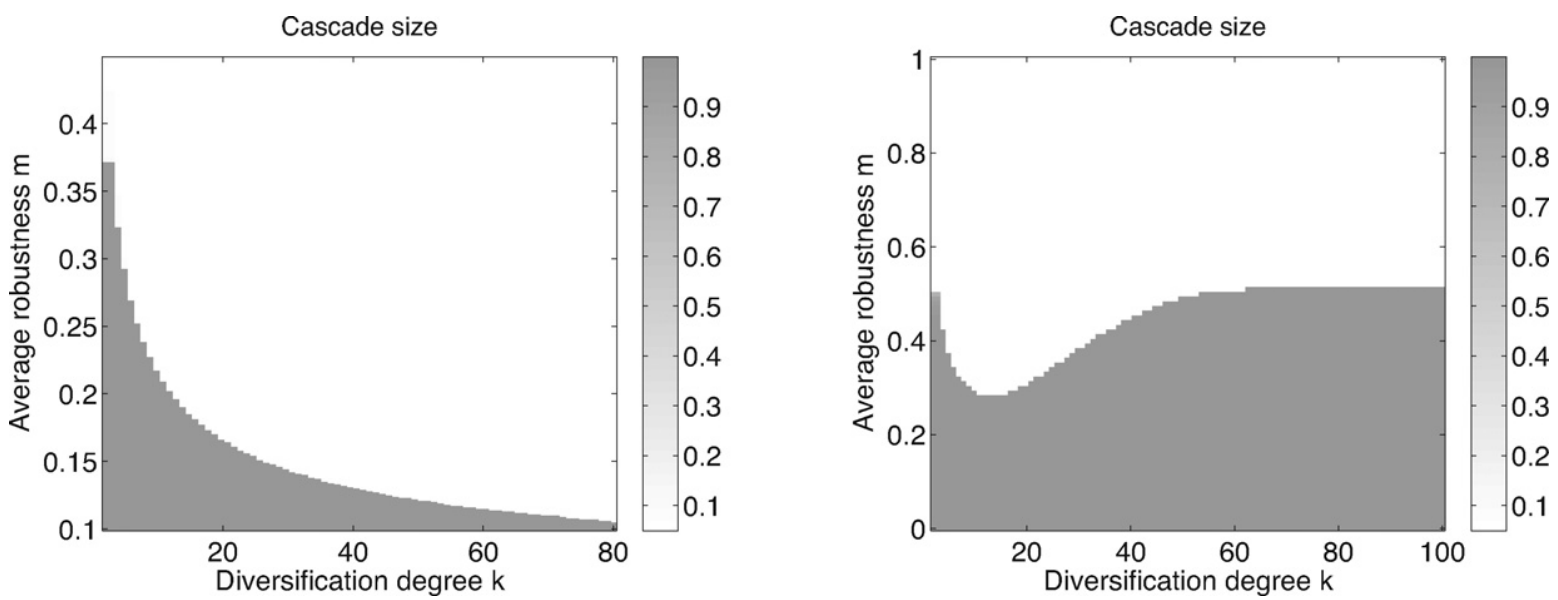

Fig. 5. Cascade size diagram (see Fig. 3). Case: $y_{0}=0, b=0, \sigma=0.3$ (left). Case: $y_{0}=0.01, b=0.4, \gamma=0.1, \sigma=0.3$ (right).
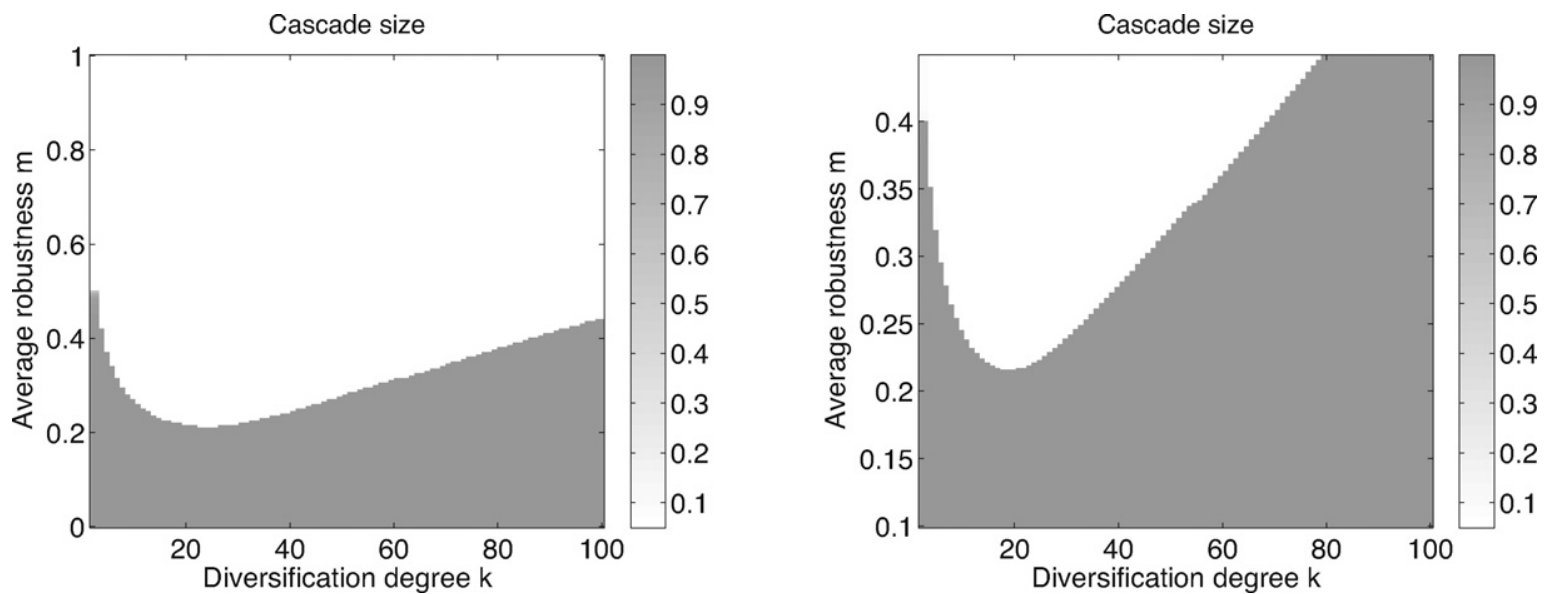

Fig. 6. Cascade size diagram (see Fig. 3). Case: $y_{0}=0, b=0.4, \gamma=0.05, \sigma=0.3$ (left). Case: $y_{0}=0.05, \gamma=0.1, b=0.5, \sigma=0.3$.

right; Fig. 6, left and right). This finding is in stark contrast with the one of the previous point and results directly from the credit runs. When agents observe that one of their obligors has a large number of defaulting counterparties, compared to her robustness, they decide to withdraw their short-term funds from her. The run imposes a cost that often pushes the agent into default, although not always. This depends on her initial level of robustness and also on the cost $b$ of the credit run (see Eq. (12)). The boundary curve separating the regime where cascades are small from where they are large has a rebound for increasing $k$. With larger values of $b$ such rebound grows steeper. This means that higher costs of credit runs imply stronger adverse effects of diversification. However, for smaller values of $\gamma$ the rebound starts only at larger values of $k$. Thus, a higher threshold for the credit run to occur implies that the adverse effect of diversification steps in only at larger values of $k$.

Scenario 7. Diversification has no effect when the system is fragile, relative to the exogenous shock. There exists a range of parameter values, i.e. $\sigma, m \in[00.4] \times[00.2]$, with $y_{0} \in[0.051], \gamma=0.1$ and $b>0.2$, where the cascade size $s$ is constant with diversification $k$. An example can be seen in Fig. 6, right) for $m<0.22$. Thus, if the average robustness is low, then the level of diversification has no effect because the systemic default occurs anyway.

Robustness of the results in case of partial asset recovery. It is interesting to discuss how the results are affected in case banks are able to recover in part the funds invested in contracts with defaulted counterparties. This corresponds to the case of the parameter $a<1$.
As shown in Fig. 7 (top left and right), in absence of runs, ${ }^{11}$ diversification is, as before, always beneficial but the cascades are reduced in size. In the presence of runs (Fig. 7, bottom left and right), even with values $a=0.25$ or $a=0.5$ the ambiguous role of diversification persists. The case $a=1$ (not shown) is very similar to $\mathrm{a}=0$. . $^{12}$

To understand the reason, notice that in Eq. (11), the parameter $a$ affects directly only the term in the dynamics that accounts for the externalities of the first type. The fact that agents recover part of the funds implies that in the face of defaulting counterparties their robustness decreases less and thus the run is less probable to occur. However, in case short-term creditors do make a run, the cost of the run is not decreased by having $a<1$, because the damage created by the run is independent of the losses due the defaulted counterprties. The cost of the run is already captured by the parameter $b$. Therefore, the overall effect of $a<1$ is to make the system more robust again large cascades when diversification is small. However, when diversification is large, then large cascades are still triggered as in the case $a=1$.

\footnotetext{
11 See the note regarding the expressions "absence of runs" or "presence of runs".

12 Of course in the case $a=0$ and with no initial shock $\left(y_{0}=0\right)$ (not shown), then no cascade occur.
} 

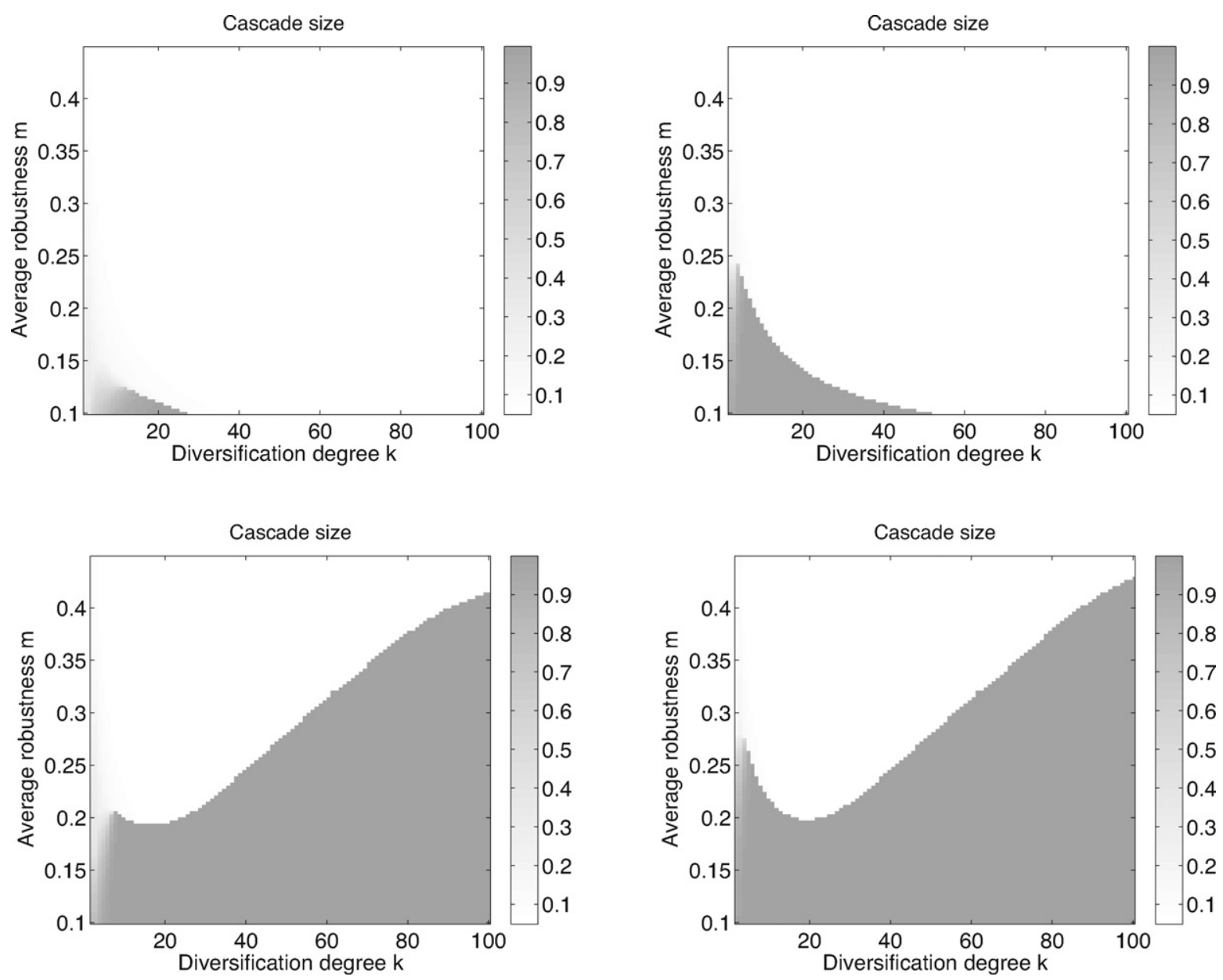

Fig. 7. Cascade size diagram (see Fig. 3). Case: $a=0.25, y_{0}=0.05, b=0, \gamma=0.05, \sigma=0.3$ (top left). Case: $a=0.5, y_{0}=0.05, \gamma=0.1, b=0, \sigma=0.3$ (top right). Case: $a=0.25, y_{0}=0.05$, $b=0.4, \gamma=0.05, \sigma=0.3$ (bottom left). Case: $a=0.5, y_{0}=0.05, \gamma=0.1, b=0.5, \sigma=0.3$ (bottom right).

\subsection{Concluding remarks}

In this paper, we contribute to the literature on the effects of risk diversification on systemic risk by developing a new model of default cascades in financial networks in which two kinds of external effects of the defaults may occur. An externality of the first type occurs when the loss incurred by an agent facing the default of a counterparty is simply proportional to the relative exposure of the agent to her counterparty. An externality of the second type, instead, depends also on the absolute number of defaults among the counterparties. In this paper, we have shown how the second type of externality may arise in presence of possible runs on the agent by her short-term lenders.

We have investigated how the number of defaults in the system depends on the diversification under various conditions. In particular we have tested how the impact of diversification depends on the average robustness of the agents, the degree of heterogeneity of financial conditions across agents (cross-sectional variance), the size of the exogenous shocks and cost of credit runs. We have shown that credit risk diversification has ambiguous results, especially in the presence of credit runs. Indeed the benefit of diluting the loss of the defaults is counterbalanced by the fact that agents are more exposed to credit runs when they have many counterparties.

This analysis contributes the following message to the debate on policies aiming at enhancing financial stability: Individual risk diversification may have ambiguous effect at systemic level. In particular, network structure and heterogeneity of levels of financial robustness across agents should be carefully taken into account when trying to devise policies that enhance the resilience of the financial system.

Our work can be extended in various directions. One line of further research we want to investigate in the future is to what extent these results are affected by assuming different network structures. Another one concerns the endogenous evolution of links over time. Finally, one could try to replicate and enrich these results in the 3dates theoretical framework that is more commonly followed in financial economics (Allen et al., 2010).

\section{Acknowledgements}

Authors acknowledge the financial support from: the ETH Competence Center Coping with Crises in Complex Socio-Economic Systems (CCSS) through ETH Research Grant CH1-01-08-2; the European Community Seventh Framework Programme (FP7/20072013) under Socio-economic Sciences and Humanities, Grant agreement no. 225408 (POLHIA); the European Commission FET Open Project FOC no. 255987; the Swiss National Science Foundation Grant no. CR12I1-127000/1); the Institute for New Economic Thinking, Grant no. IN01100028INET "Financial Fragility and Systematic Risk. Policy Implications of Heterogeneous Agents Models". 


\section{Appendix A.}

Proof of Proposition 1. We ask what is the fraction $s$ of nodes that have failed so far at time $t$. This is

$s(t+1)=\operatorname{Pr}\left\{\eta_{i}(t+1)<0\right\}=\operatorname{Pr}\left\{\sum_{j=1}^{k} \chi_{j}(t)>\eta_{i}(0)\right\}$.

For simplicity, we assume the network is a regular graph with degree $k$. Whether a given node $i$ fails by the time step $t+1$, it depends on the number $k_{f}$ of the neighbors that have already failed, out of the total number $k$ of neighbors. Denote $\chi_{j}(t)=1$ iff $j$ has defaulted any time before or at $t$, and 0 otherwise. Then, at time step $t+1$, the possible events are $k_{f}=\sum_{j=1}^{k} \chi_{j}(t)=1,2, \ldots, k$. In each of these events, the probability that node $i$ fails, depends on the initial value of its robustness. Assume all failures that have occurred so far are uncorrelated across agents. Then, they follow a binomial distribution, $\mathrm{P}_{r}\left\{k_{f}\right.$ failures among $k$ neighbors $\}=$ $\left(\begin{array}{c}k \\ k_{f}\end{array}\right) p^{k_{f}}(1-p)^{k-k_{f}}$, where $p$ is the probability that any given node has failed so far. In the limit of a large network it is $p=n(t)$. Finally, we need to take into account that firms do not recover during the cascade and thus the fraction of failure can only increase. Therefore,

$$
\begin{aligned}
s(t+1)= & \max \left\{s_{0}, \sum_{k_{f}=1}^{k}\left(\begin{array}{c}
k \\
k_{f_{i}}
\end{array}\right) s(t)^{k_{f}}\right. \\
& \left.\times(1-s(t))^{k-k_{f}} \operatorname{Pr}\left\{\eta_{i}(t) \leq \frac{a k_{f}}{k}\right\}\right\},
\end{aligned}
$$

where $s_{0}$ is the initial fraction of failures. This is a recursive equation of the type $s(t+1)=F(s(t))$. Once the probability distribution of $\eta$ is specified, the fixed points are the solutions of $s=F(s)$. For example, in the case of regular graph, with $\eta$ following a Gaussian distribution with mean $m$ and standard deviation $\sigma$ we obtain:

$$
\begin{aligned}
s(t+1)= & \max \left\{s_{0}, \quad \sum_{k_{f}=1}^{k}\left(\begin{array}{c}
k \\
k_{f}
\end{array}\right) s(t)^{j}(1-s(t))^{k-k_{f}} \frac{1}{\sigma \sqrt{2 \pi}} \int_{-\infty}^{a k_{f} k}\right. \\
& \left.\exp \left(-\frac{\left(u-m^{2}\right)}{2 \sigma^{2}}\right)\right\}=\max \left\{s_{0}, \sum_{k_{f}=1}^{k}\left(\begin{array}{c}
k \\
k_{f}
\end{array}\right) s(t)^{k_{f}}\right. \\
& \left.\times(1-s(t))^{k-k_{f}} \frac{1}{2}\left(1+\operatorname{erf}\left(\frac{a k_{f} / k-m}{\sigma \sqrt{2}}\right)\right)\right\}
\end{aligned}
$$

Notice that $F(s)>s_{0}$ with $s_{0}>0$ strictly for $m<\infty$ and $\sigma>0$. Since in addition $F(s)$ is not decreasing, there exists at least one stable fixed point. There maybe more than one but what matters here is only the smallest stable fixed point $s$ with $s \geq \mathrm{S}_{\circ}$. The Equation above can be solved numerically with arbitrary precision for any choice of the parameters. The results are shown in Fig. 1.

In general it would also be possible to account for heterogeneous degree distribution. This requires however a more extended analytical treatment that goes beyond the objective of this paper. $\square$

Proof of Proposition 2. The procedure is the same as in the Proof of Proposition 1 with the difference that the probability that agent $i$ 's robustness goes below the threshold depends not only on the direct loss due to the defaulting counterparties but also on whether the credit run occurs or not. Since the credit run occurs iff the robustness is smaller than the number of defaulted counterparties time the factor $\gamma$, we obtain

$P\{$ agent $i$ defaults $\}=$

$P\left\{\eta_{i}(t) \leq \frac{a k_{f}}{k}\right\} P\left\{\eta_{i}>\gamma k_{f}(t)\right\}+P\left\{\eta_{i}(t) \leq \frac{a k_{f}}{k_{i}}+b\right\} P\left\{\eta_{i} \leq \gamma k_{f}(t)\right\}$.

By replacing the expression above in Eq. (A.1) we obtain the first point of the proposition. The existence of the solution follows as in the previous proof. $\square$

\section{References}

Acharya, V.V., 2009. A theory of systemic risk and design of prudential bank regulation. Journal of Financial Stability 5 (3), 224-255, URL: http://www.sciencedirect.com/science/article/pii/S1572308909000059.

Allen, F., Gale, D., 2000. Financial contagion. Journal of Political Economy 108 (1), 1-33.

Allen, F., Babus, A., Carletti, E., 2010. Financial connections and systemic risk. Economics Working Papers.

Amini, H., Cont, R., Minca, A., 2010. Stress Testing the Resilience of Financial Networks, URL: http://ssrn.com/paper=1730732.

Bartram, S.M., Brown, G.W., Hund, J.E., 2007. Estimating systemic risk in the international financial system. Journal of Financial Economics 86 (3), 835-869, URL: http://www.sciencedirect.com/science/article/B6VBX4P40KT1-1/2/077862527602.

Battiston, S., Gatti, D.D., Gallegati, M., Greenwald, B.C.N., Stiglitz, J.E., 2009. Liaisons dangereuses: increasing connectivity, risk sharing, and systemic risk. NBER Working Paper w 15611.

Boss, M., Summer, M., Thurner, S., 2004. Contagion flow through banking networks. Lecture Notes in Computer Science 3038, 1070-1077.

Brunnermeier, M., 2008. Deciphering the 2007-08 liquidity and credit crunch. Journal of Economic Perspectives 23 (1), 77-100.

Brunnermeier, M., Pederson, L., 2009. Market liquidity and funding liquidity. Review of Financial Studies 22, 2201-2238.

Callaway, D., Newman, M., Strogatz, S., Watts, D., 2000. Network robustness and fragility: percolation on random graphs. Physical Review Letters 85 (25), 5468-5471.

Castiglionesi, F., Navarro, N., 2010. Optimal fragile financial networks. SSRN Working Paper Series. URL: http://ssrn.com/paper=1089357.

Christian, Upper, 2011. Simulation methods to assess the danger of contagion in interbank markets. Journal of Financial Stability 7 (3), 111-125, URL http://www.sciencedirect.com/science/article/pii/S1572308910000550.

Cont, R., Moussa, A., Santos, E.B., 2010. Network Structure and Systemic Risk in Banking Systems, URL: http://ssrn.com/paper=1733528.

Eisenberg, L., Noe, T., 2001. Systemic risk in financial systems. Management Science 47 (2), 236-249.

Elsinger, H., Lehar, A., Summer, M., 2006. Risk assessment for banking systems. Management Science 52 (9), 1301-1314.

Frey, R., McNeil, A., 2003. Dependent defaults in models of portfolio credit risk. Journal of Risk 6, 59-92.

Furfine, C., 2003. Interbank exposures: quantifying the risk of contagion. Journal of Money Credit \& Banking 35 (1), 111-129.

Gai, P., Kapadia, S., 2010a. Contagion in financial networks. Bank of England Working Papers.

Gai, P., Kapadia, S., 2010b. Liquidity hoarding, network externalities, and interbank market collapse. Bank of England Working Papers.

Haldane, A., 2009. Why banks failed the stress-test.

Iori, G., Jafarey, S., Padilla, F., 2006. Systemic risk on the interbank market. Journal of Economic Behaviour and Organisation 61 (4), 525-542.

Kleinberg, J., 2007. Cascading behavior in networks: algorithmic and economic issues. Algorithmic Game Theory.

Rochet, J., Vives, X., 2004. Coordination failures and the lender of last resort: was Bagehot right after all? Journal of the European Economic Association 2 (6), 1116-1147.

Shin, H., 2008. Risk and liquidity in a system context. Journal of Financial Intermediation 17, 315-329.

Stiglitz, J., 2010. Risk and global economic architecture: why full financial integration may be undesirable. NBER Working Paper.

Wagner, W., 2010. Diversification at financial institutions and systemic crises. Journal of Financial Intermediation 19 (3), 373-386, Risk Transfer Mechanisms and Financial Stability. URL: http://www.sciencedirect.com/science/article/B6WJD4WW9KSD-1/2/a49b0c731639. 